\title{
Effect of the Error Propagation Rates in Developing a Skin Model of Measured Surfaces in Coordinate Metrology
}

\author{
Cody Berry, Lillian Goodwin, and Ahmad Barari \\ Faculty of Engineering and Applied Science \\ UOIT \\ Oshawa, Canada \\ ahmad.barari@uoit.ca
}

\begin{abstract}
Coordinate metrology is the most commonly used inspection method to analyze surfaces for manufacturing errors and imperfections. The computational in today's coordinate metrology are composed of a series of extensive computational tasks. Developing a triangulated skin model using a finite element method is an approach to construct a surface based on the discrete measured points, which can be used for integrated computational algorithms reducing the uncertainty of the inspection process. Reducing the errors in developing the skin model by calibrating the two main parameters of the algorithm, which specify the propagation of the geometric deviations in two orthogonal directions, is the objective in this work. By varying theses propagation parameters and tracking the overall error of the constructed skin model, their effects are evaluated. Various cases are studied and the resulting patterns of the errors for different surfaces are used to make suggestions on selection of the two parameters resulting accurate construction of the skin model of the measured work pieces based on the coordinate metrology data. This will provide a reliable technique to develop the skin model of the manufactured products for quality control, manufacturing process control, or repair and finishing of the products.
\end{abstract}

Keywords: coordinate metrology; skin model; error propagation rates; Deviation Zone Evaluation (DZE); Point Measurement Planning (PMP)

\section{INTRODUCTION}

Coordinate metrology incorporates the use of three different processes, namely, Point Measurement Planning (PMP), Substitute Geometry Estimation (SGE), and Deviation Zone Evaluation (DZE). In such systems, the inspection accuracy is subject to the issue of uncertainties due to estimating some aspects of a probability distribution on the basis of sampling which adversely affects the accuracy and reliability of the inspection.

Selection of location, distribution, and number of sample points using the available information is the objective of PMP

Sponsored by NSERC process. Finding the best ideal substitute geometry that represent the selected sample points using a desired fitting criteria is performed by SGE process. Developing a comprehensive representation of geometric deviation for any point of the measured surface including the points that are not physically measured, is the goal of DZE process. These three computational tasks were traditionally conducted in a sequential manner. All three tasks have access to three sets of external data including: the desired geometric features which are typically represented by Computer Aided Design (CAD) models, data and information from the corresponding manufacturing operations used to fabricate the work piece, and the objective of the inspection process corresponding to the downstream processes that are planned for the measured work piece [1].

Output of each task is used as the input for the next task in the PMP-SGE-DZE sequential order. The typical output of the measurement instruction developed by PMP is used for capturing a limited number of discrete $3 \mathrm{D}$ points from the work piece. This data set is used as an input to SGE to fit the best representative substitute geometry. The external data regarding the objective of the inspection process is used to define the fitting criteria in SGE process. The geometric deviations of the measured sample points are calculated using the results of SGE and are input to the finishing computational task, i.e., DZE. DZE is responsible to analyze the distribution of geometric deviations and represent a detailed deviation zone for the entire measured surface. This result is the ultimate output of the coordinate metrology process which can be represented by statistical characteristics of the distribution and/or a full skin model of the measured surface. It is worth mentioning that, in order to develop the full skin model, DZE should be capable to estimate the geometric deviation function for the entire measured surface including infinite points that are not physically measured [1].

The concept of skin model is introduced in the field of tolerance analysis [2]. Tolerances are specified during the design process to meet the desired form, fit, and functionality of the products. Today's design and manufacturing paradigms do not allow the design tolerance analysis being performed without considering the manufacturing uncertainties. A 
detailed representation of the geometric deviations from the best fitted desired geometry, which are mainly caused by the inherent errors in the employed manufacturing processes is needed to complete an integrated tolerance analyses. This detailed model of geometric deviations is a non-ideal Surface, which is also referred as a Skin Model of the part in Geometrical Product Specification (GPS) standards [2]. The need for developing a Skin Models is addressed by the standards and is the new focus of research [3]. However, methodologies to develop these models need further research.

A skin model is a discretized surface generated from a point cloud. The advantage of this method is its ability to reflect the deviation of the surface at any location, even those that were not sampled from. This data can be used to allow for virtual sampling of the piece. In a previous work, Lalehpour and Barari developed a finite element method to develop the skin model using the coordinate metrology discrete $3 \mathrm{D}$ points [1]. However, the developed method was not fine-tuned to provide the most accurate results. This paper will seek to finetune that method. The accuracy of the developed skin model is of paramount importance in design and manufacturing systems. The skin model is used directly for any possible downstream design and manufacturing processes that need the results of coordinate metrology process, this includes planning for precise finishing operations, manufacturing error compensation, closed-loop of manufacturing and inspection, dynamic process control, or for any tolerance evaluation required for the assembly and interaction of the work piece with the other mechanical components. Therefore, the added value in developing an accurate skin model of the work piece to the design and manufacturing processes is highly significant.

It is shown in several recent research [4-8] that integration of PMP, SGE, and DZE tasks will result in reducing the computational uncertainties in coordinate metrology. Another interesting application of a full skin model is to reduce the uncertainties of coordinate metrology process by conducting mutual closed loops between the computational tasks. The developed skin model by DZE is dynamically feed-backed to SGE and PMP to improve the processes in integrated inspection system using DZE-SGE and DZE-PMP closedloops.

\section{THEORETICAL BACKGROUND}

Lalehpour and Barari [1] developed the finite element method utilized in this paper. In this method, the data set that is obtained from the surface is first randomly pruned down. This is done as the entire sample set should not be required to accurately create a skin model. These points that are removed from the skin model data set will be used to validate the skin model once it is generated.

The new sample set is then transformed from real space, where $\mathrm{x}, \mathrm{y}$ and $\mathrm{z}$ coordinates are used, to a parametric space. In this space, $\mathrm{u}, \mathrm{v}$, and $\mathrm{d}$ become the axes. The two largest principal axes of the data set become the $u$ and $v$ axes, while the smallest principal axis becomes $d$, or the geometric deviations. The data set is then divided into windows. Each of this windows is equal in size, and a set number of points based on the size of the data set is selected from each window. These will become non-moving vertices of the skin model. This skin model relies on triangular elements, so a Delaunay triangulation is used on these non-moving vertices to create the triangular model.

This triangular surface becomes the basis for the rest of the skin model. The areas of each triangle are checked for size, with large areas being subdivided into smaller ones with the addition of another point at the centroid. This process repeats until all triangles are under the size threshold defined by the overall size of the data set. This increase in density causes an increase in accuracy for the finished skin model. These additional points do not initially have the correct geometric deviation to accurately represent the measured surface. In order to determine the correct placement of these vertices, a finite element method was used using Poisson Equation as follows:

$$
\frac{\partial}{\partial u}\left(K_{u u} \frac{\partial S}{\partial u}\right)+\frac{\partial}{\partial v}\left(K_{v v} \frac{\partial S}{\partial v}\right)+E=0
$$

where, $\mathrm{u}$ and $\mathrm{v}$ are two orthogonal directions on the measured surface, $\mathrm{K}_{\mathrm{uu}}$ and $\mathrm{K}_{\mathrm{vv}}$ are the error propagation rates in $\mathrm{u}$ and $\mathrm{v}$ directions, $\mathrm{E}$ is the determined geometric deviation, and $\mathrm{S}$ is the skin model. The $\mathrm{K}$ in [1], these $\mathrm{K}$ values were both assumed to be equal to unity for simplification and validation purpose. This paper will focus on determining the optimal $\mathrm{K}$ values. The effect of these two parameters on the accuracy of the skin model is studied in this paper. This resulting information can be used to produce more accurate skin model or to be used as the initial condition for an optimization process. Particle swarm optimization algorithm [9] is suggested for this case which is one of many algorithms that optimize based on different patterns found in nature.

\section{METHODOLOGY}

A large amount of data is measured from the work-pieces using a coordinate measuring machine (CMM). The PMP process is then deployed to virtually sample from the given datasets. Once the smaller datasets were chosen, SGE is performed to find the closest representative plane to fit the given dataset. The method utilized for this purpose is total least squares (TLS) fitting to find the best fit plane for the given surfaces. This can be utilized for the SGE process because of the use of solely planar geometry.

The propagation rates of geometric deviations (PRGD), both in the $\mathrm{u}$ and $\mathrm{v}$ directions, used to construct the skin model are examined using the following process. A 40 x 40 matrix of PRGD values in the $u$ and $v$ directions ranging from -20 to 20 discrete values. This constructs a total of 1600 different skin models for each given dataset. After the skin model is constructed and the deviation of each point is known, the total error for each skin model is calculated by taking the difference from the generated original chosen data points to the generated skin model. The total error is the cumulative sum of square of the errors for each skin model. 
The effect of the fitting parameters (PRGDs) is analyzed by graphing the total error for each skin model to show the total error for the given matrix of values. The results are then optimized using an algorithm to find the lowest values and removing them recursively to find a trend line of values. This trend line is then analyzed for patterns involving different topologies.

\section{RESUlTS AND DISCUSSION}

In order to determine the area for optimization, the results of the error value for the 1600 skin models was graphed for each case study. Since the observed pattern were consistent, the graphs corresponding a typical case study is used for the rest of this paper.

Fig. 1 presents the error values corresponding to 1600 skin models developed for the case study, with a random sample piece for illustrative purposes. With this data, it was easy to determine problem values for the error propagation rate values. The worst results were consistently those where the error propagation rates had signs that differed from one another. With this in mind, a filter was applied to remove a large number of the error samples. For the first pass, any value larger than $0.000001 \%$ of the minimum value was removed. This resulted in Fig. 2.

In Fig. 2, the sections with the smallest error can be seen. Those areas where the error propagation rates had differing signs have been completely removed. Another interesting characteristic is the symmetry developed between the two remaining segments. This means the optimization algorithm can be configured to only search one small segment of the overall data set, and it will be likely to achieve an acceptable result in a short amount of time.

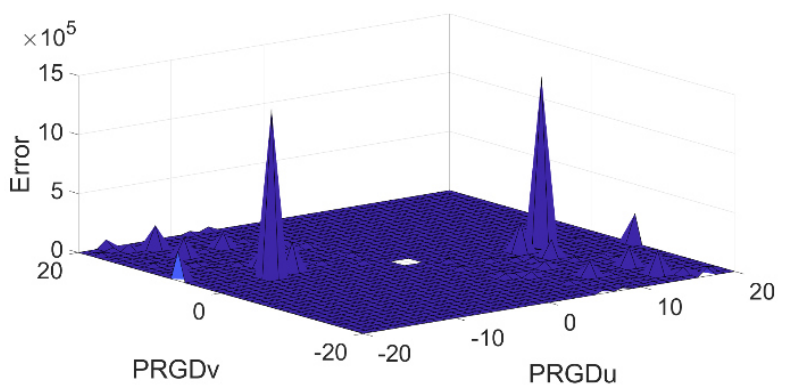

Figure 1. Error Results for 1600 Skin Models

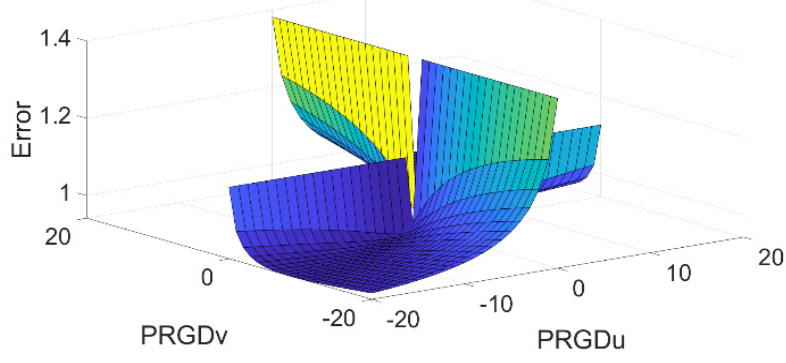

Figure 2. $\quad 99.999999 \%$ of the Range Removed

Another factor looked at was the density of points in different locations. This was examined to see if there was a correlation between high density zones of points and the lowest error results. With this information, the optimization area could be further refined. To test this, a data set was generated with equally spaced points. Then, a section of the data set was populated with a much higher density data set. Finally, the error graph generated was examined to determine where the lowest points were found. These points had a line fit to them, and the slope of the line was examined. Fig. 3 shows the first of these data sets.

Data Set 1 is heavily weighted to the positive $\mathrm{x}$ direction, and is shown in Fig. 3. Fig. 4 shows the results of the error analysis. The final graph has the errors of the opposing signed error propagation rates removed like in Fig. 2. There is also a large decrease in error between these two figures as data set 1 is very planar. None of the minimum error values were found in either of those regions, which was to be expected.

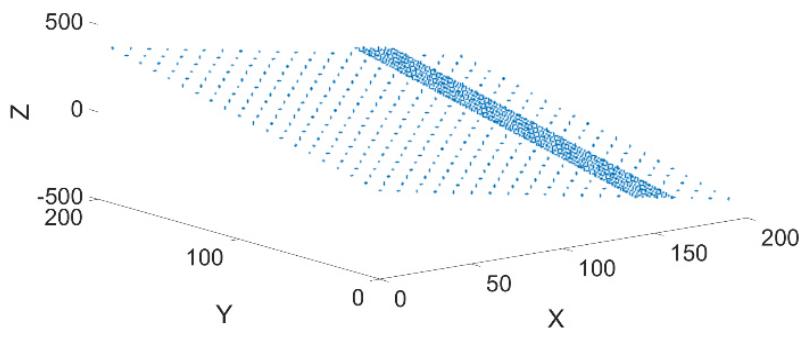

Figure 3. Data Set 1

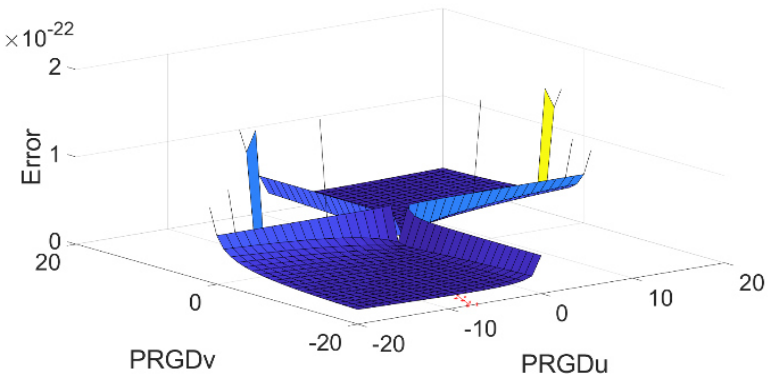

Figure 4. Data Set 1 - Error Modeling Results 
The red line on the bottom of the graph is the best fit line for the lowest error values. It has a very large slope, which shows that the error propagation rate in the $\mathrm{v}$ axis on the refit data set dominates, corresponding with the increase in point density. This result is reflected in Fig. 5 and Fig. 6.

As the high density area is moved along the $\mathrm{x}$-axis in the negative $\mathrm{x}$ direction, there is a corresponding shift in the slope of the line in the results. This test was repeated with the high density section moving along the entire u-axis. In each case, the slope of the line continued to shift in the same direction. This meant that the location of the points had an effect on the location of the minimum values, and therefore the start conditions for the optimization algorithm could be selected more intelligently, increasing the accuracy of the skin model as it would be more likely to find a better solution.

\section{CONCLUSION}

The goal of this paper was to determine the area of optimization for the two main parameters involved in the skin model process, i.e. the geometric error propagation rates in the two orthogonal directions. A series of tests are conducted to model the effect of the error propagation rates on the accuracy of the resulting skin model. It was found that when the PRGD values had different signs from each other, the error was greatly increased. This allowed for the removal of those regions from analysis. Further examination showed that the remaining regions were symmetric, and therefore only one of them needed to be used to find the best PRGD values. Next, the effect of the density of sample points on the error values was examined.

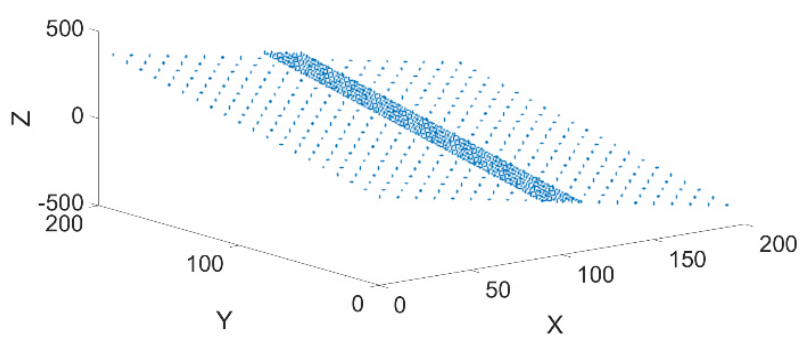

Figure 5. Data Set 2

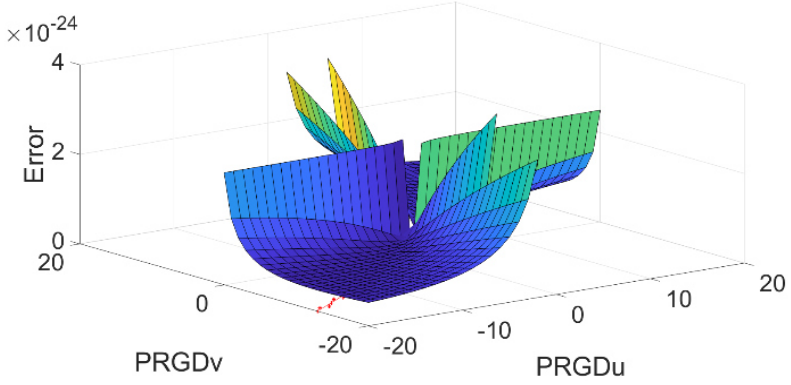

Figure 6. Data Set 2 - Error Modeling Results
The results showed that this density did have a large effect on the error. This effect was also fairly systematic, and therefore could be predicted. With this information, the area of optimization can be further refined. Both of these results can be used to better customize the skin model algorithms and achieve more accurate results using the skin model method.

\section{ACKNOWLEDGMENT}

The research support provided by the Natural Science and Engineering Research Council of Canada (NSERC) is greatly appreciated.

\section{REFERENCE}

[1] A. Lalehpour and A. Barari, "Developing skin model in coordinate metrology using a finite element method," Measurement, vol. 109, pp. 149-159, 2017.

[2] EN ISO 17450-1:2011, Geometrical product specifications (GPS) General concepts - Part 1: Model for geometrical specification and verification (2011).

[3] B. Schleich, N. Anwer, L. Mathieu, S. Wartzack, "Skin model shapes: A new paradigm shift for geometric variations modelling in mechanical engineering", Computer-Aided Design, Vol. 15, pp. 1-15, 2014.

[4] A. Barari, S. Jamiolahmadi, "Convergence of a finite difference approach for detailed deviation zone estimation in coordinate metrology" , Acta IMEKO, Vol. 4, No 4, pp. 20 - 25, 2015.

[5] A. Barari, F. Kaji,"The uncertainty of coordinate metrology as a function of sampling strategy", Journal of CMSC, Vol. 9, No 1, pp. 2227, 2014.

[6] Jamiolahmadi, S., Barari, A., "Study of detailed deviation zone considering coordinate metrology uncertainty", Measurements, Inpress 2016, DOI: https://doi.org/10.1016/j.measurement.2016.12.032

[7] H. Gohari, A. Barari, "A quick deviation zone fitting in coordinate metrology of NURBS surfaces using principle component analysis", Measurements, https://doi.org/10.1016/j.measurement.2016.05.050

[8] A. Barari, "Inspection of the machined surfaces using manufacturing data", Journal of Manufacturing Systems, Volume 32, Issue 1, January 2013, Pages 107-113, https://doi.org/10.1016/j.jmsy.2012.07.011

[9] T. Zeugmann et al., "Particle swarm optimization," in Encyclopedia of Machine Learning, Boston, MA: Springer US, 2011, pp. 760-766. 